\section{Akne: Ist Schokolade wirklich schuld?}

Fast $60 \%$ der Heranwachsenden geben an, Akne zu haben, so das Ergebnis einer Online-Befragung mit mehr als 10.000 Teilnehmern. Aus der Analyse geht außerdem hervor, dass die Wahrscheinlichkeit, an Akne zu erkranken, um das Achtfache erhöht ist, wenn bereits Mutter und Vater Akne hatten. Ein höheres Risiko besteht offenbar auch bei Verzehr von viel Schokolade. Eine inverse Assoziation stellten die Ärzte dagegen im Zusammenhang mit dem Alter und dem Rauchverhalten fest: 21- bis 24-Jährige hatten im Vergleich zu 15- bis 17-Jährigen ein um 27\% geringeres Aknerisiko, Raucher ein um fast $30 \%$ geringeres.

Wolkenstein, Pet al. JEADV 2017 (online first)

\section{Auch Privatpatienten müssen warten}

Privatpatienten sind dabei, ihre Privilegien in den Praxen niedergelassener Ärzte zu verlieren. Die Wartezeiten von gesetzlich und privat versicherten Patienten auf Termine in Deutschland gleichen sich an. Das geht aus der aktuellen Versichertenbefragung der Kassenärztlichen Bundesvereinigung hervor. Die Gründe liegen wohl gleichermaßen in der Demografie wie der Praxisorganisation. Die Privatversicherten würden älter und benötigten häufiger ärztliche Hilfe. Gleichzeitig weiteten die Ärzte ihre Terminkontingente für Kunden der privaten Assekuranz nicht aus.

www.kbv.de

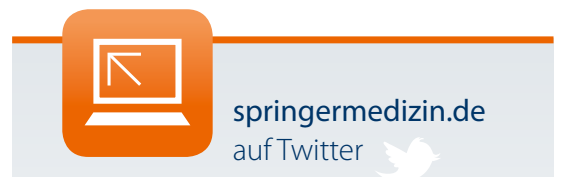

Auch im Herbst heißt es wieder:

Lauschen Sie unserem Zwitschern und werden Sie zum Follower.

- twitter.com/springermedizin

17 Wissenschaftler fordern:

„Weg mit dem

Heilpraktikerberuf!"

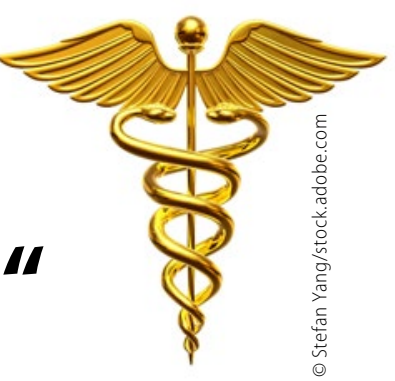

Der neu gegründete "Münsteraner Kreis", eine Gruppe von 17 Wissenschaftlern um die Medizinethikerin Bettina Schöne-Seifert, fordert aktuell das Ende für die „unangemessene Ausbildung und die meist unhaltbaren Krankheitskonzepte" der Heilpraktiker.

Der Münsteraner Kreis verweist auf einen konsentierten Vorstoß des Deutschen Ärztetages. „Vom derzeit zulässigen Tätigkeitsumfang von Heilpraktikern sind alle invasiven Maßnahmen (wie chirurgische Eingriffe, Injektionen und Infusionen) sowie die Behandlung von Krebserkrankungen auszuschließen“, forderte der 120. Deutsche Ärztetag in Freiburg in einer Entschließung zur Re- form des Heilpraktikerwesens. Der Ärztetag verband das gleichzeitig mit der Forderung an den Gesetzgeber, „eine Definition der Heilkunde an geeigneter Stelle gesetzlich zu verankern, die eine deutlichere Abgrenzung von Paramedizin und dem Heilpraktikerwesen erlaubt.“ Um eben diese Abgrenzung geht es auch den Vertretern des Münsteraner Kreises, der gar von Parallelwelten - der Welt der akademischen Medizin und der Welt der Heilpraktiker - spricht. Während die akademische Medizin nach Evidenzbasierung und begründetem Fortschritt strebe, seien Heilpraktiker in der, wie die Kritiker betonen, überwiegend unwissenschaftlichen Gedankenwelt der Komplementären und Alternativen Medizin (KAM) verankert. Matthias Wallenfels

Kein gutes Zeichen!

\title{
Wenn Dicke mit Darmkrebs abspecken
}

Nicht nur ein rascher, sondern auch ein langfristiger Gewichtsverlust kennzeichnet eine ungünstige Darmkrebsprognose - vor allem bei dicken Patienten. Nehmen sie über fünf Jahre hinweg ab, ist ihre Lebenszeit deutlich verkürzt.

Verlieren schwer kranke Patienten rasch an Gewicht, ist das in der Regel ein schlechtes Zeichen: Nicht selten klopft dann bereits der Tod an die Tür. Doch auch ein schleichender Gewichtsverlust markiert offenbar ein nahes Ende - zumindest bei dicken Darmkrebskranken. Wer über fünf Jahre hinweg zunehmend magerer wird, hat deutlich schlechtere Chancen, die kommenden Jahre zu überleben, als Patienten, die ihr Gewicht halten oder sogar noch zulegen: Die Sterberate ist dann rund doppelt so hoch. Darauf deutet eine Analyse des "Colon Cancer Family Registry (CCFR)“ mit insgesamt 2049 Teilnehmern hin. Die Patienten wurden während der ersten zwei Jahre nach ihrer Krebsdiagnose zu dem Gewicht vor der Diagnose befragt. Im Median fünf Jahre nach der Diagnose wurden die Überlebenden erneut befragt. Fünf Jahre nach der Diagnose hatten 19\% der Patienten mindestens $5 \%$ ihres Körpergewichts verloren, $48 \%$ blieben stabil und 33\% hatten mindestens $5 \%$ zugelegt. Im zehnten Jahr nach der Diagnose waren 344 Patienten gestorben. Von den Patienten mit Gewichtsverlust starben bis zum zehnten Jahr $30 \%$. Jeweils $14 \%$ und $13 \%$ waren es bei Darmkrebskranken mit stabilem und gesteigertem Gewicht.

Thomas Müller 